\title{
LA TUTORÍA Y LA ORIENTACIÓN UNIVERSITARIA EN LA NUEVA COYUNTURA DE LA ENSEÑANZA SUPERIOR: EL PROGRAMA "VELERO"
}

\author{
Pedro R. Álvarez Pérez \\ Universidad de La Laguna
}

\begin{abstract}
RESUMEN: Este artículo aborda el tema de la tutoría universitaria, enmarcándola en la coyuntura actual de la enseñanza superior, donde se vive una sucesión casi continua de movimientos y cambios que emanan básicamente de los acuerdos de Bolonia. En este mar de turbulencias, parece que una de las tendencias a seguir apunta al énfasis que quiere ponerse en los procesos de aprendizaje del alumnado y en la labor tutorial que debe realizar el profesorado universitario. Para facilitar esta tarea, se ha diseñado el programa $V E L E R O$, con el que se pretende facilitar los procesos de adaptación y desarrollo personal, académico y profesional del alumnado de nuevo ingreso.
\end{abstract}

ABSTRACT: This article approach tutoring at the University level in the context of the rapid changes that are undergoing European Universities as a result of the Bolonia Agreements. In these troubled waters, it seems that one of the main trends points to the relevance attached to students learning processes and the correponding tutorial role of University teachers. To facilitate the enacting of that role we have designed the VELERO program, in order to support and foster the processes of adaptation and professional, academic and personal development of first year university students.

PALABRAS CLAVE: Orientación Universitaria; necesidades de orientación; función tutorial del profesorado.

KEY WORDS: Guidance university; guidance needs; teachers tutorial function.

\section{INTRODUCCIÓN}

Los cambios que se están experimentando en la enseñanza superior, los fines que se persiguen con la misma, las demandas que la sociedad plantea y la problemática a la que se enfrenta diariamente el alumnado que cursa estudios universitarios, ha llevado a que en los últimos tiempos se hayan potenciado los servicios encarga- 
dos de desarrollar acciones orientadoras en este tramo de la enseñanza y se haya comenzado a resaltar la figura del profesor tutor (Álvarez y Cabrera, 1998; Álvarez y Lázaro, 2002; Álvarez y Jiménez, 2003).

La necesidad de que el profesorado se implique en la labor orientadora es evidente si se tiene en cuenta, no sólo la gran cantidad de estudiantes que pueblan las aulas durante los primeros cursos y que hacen casi imposible una ayuda personalizada del docente de cada materia, sino también la gran heterogeneidad de éstos. Este alumnado, al contrario de lo que parece, no ha alcanzado el grado de madurez que le haga autónomo e independiente para hacer frente a la diversidad de situaciones nuevas a las que debe dar respuesta, por lo que sin una ayuda apropiada, corre el riesgo de perderse en el camino de la transición, prolongar o abandonar los estudios. Saldaña (1986:272), haciéndose eco de esta situación, dice que uno de "los problemas más graves de la Universidad Española es el porcentaje de alumnos que abandonan sus estudios".

Efectivamente, en la coyuntura actual en la que se aprecia un descenso demográfico considerable que comienza a afectar la matrícula de algunas titulaciones universitarias, la situación de fracaso académico, las tasas de abandono, el número de repetidores, etc. pasa a ser un tema importante, imponiéndose la necesidad de encontrar vías que lleven a incrementar los resultados positivos de este criterio de evaluación institucional y que hagan disminuir estos porcentajes de fracaso y abandono. Lo mismo sucede con el problema de la permanencia, ya que la prolongación de la estancia de los estudiantes acarrea un coste adicional a la Universidad: se masifican las aulas con los alumnos repetidores, se generan más grupos con lo cual se necesita más profesorado, se reduce la eficacia de los Servicios, etc.

Por estas y otras muchas razones la enseñanza universitaria se ha convertido en un nivel educativo que reclama con insistencia la atención necesaria para que se introduzcan las medidas oportunas que eviten o palien el efecto de factores que interfieren en el normal desarrollo académico del alumnado. Entre estas medidas, la acción tutorial constituye una importante iniciativa encaminada a asegurar la retención del alumnado y a mejorar el proceso de aprendizaje, que la mayoría de las universidades han introducido como actividades de apoyo al proceso educativo. En este trabajo se presenta el programa velero, un plan de actividades tutoriales adaptado al nivel universitario, preferentemente para el alumnado que se incorpora por primera vez a la vida universitaria, cuya finalidad es facilitar la adaptación y el proceso de aprendizaje.

\section{IMPORTANCIA DE LA TUTORÍA Y LA ORIENTACIÓN EN EL CONTEXTO UNIVERSITARIO}

Son numerosos los estudios en los que se pone de manifiesto las necesidades de información y de orientación del alumnado inmerso en procesos de transición y adaptación a la enseñanza universitaria (Díaz, 1989; Castellano y Sanz, 1990; Arbizu, 1994; Martín, Moreno y Padilla, 1998). En una investigación realizada en la Facultad de Educación de la Universidad de La Laguna (ULL) al comienzo del curso 1997-98 con una muestra de 417 estudiantes que realizaban estudios de primer 
curso en las distintas titulaciones que se ofertaban en el mismo (Álvarez, Cabrera, Feliciano y Santana, 1999), se encontró que :

$\checkmark$ El 86\% manifestó poseer poca o ninguna información previa acerca del contenido de las asignaturas a cursar.

$\checkmark$ El $88.8 \%$ desconocía el tipo de prácticas que se realizan.

$\checkmark$ El 57\% desconocía las salidas profesionales.

$\checkmark$ El 40.8\% no sabía el número de créditos a cursar.

$\checkmark$ El 91.7\% no tenía información sobre los itinerarios formativos.

La atención a estos problemas que expresa el estudiantado (escaso conocimiento de los estudios, poca orientación recibida, dificultades para adaptarse al nuevo espacio vital, diferencias en el proceso de enseñanza/aprendizaje, baja motivación, etc.) ha hecho que la acción orientadora universitaria haya comenzado a emerger con una enorme vitalidad en los últimos tiempos. Y, como consecuencia, los procesos de tutorización, orientación e información al alumnado se han empezado a contemplar como un indicador importante de calidad de las universidades, lo que ha derivado en la puesta en marcha de una diversidad de iniciativas (jornadas de acogida y bienvenida a los estudiantes de nuevo ingreso, utilización de métodos para fomentar el aprendizaje autónomo del alumnado, actividades para compensar dificultades académicas o lagunas formativas, etc.).

Hablar de educación universitaria supone actualmente contemplar, además de los contenidos académicos y cognoscitivos, todos aquellos que pertenecen a esa otra esfera de lo personal, social y profesional. Lo preocupante es que en muchos casos han quedado olvidados y sin atender, al menos de manera sistemática, ordenada y planificada, muchos contenidos que en un futuro más o menos cercano se convertirán en exigencias que el mundo empresarial propondrá a las instituciones universitarias. Nos referimos al cultivo de esas otras competencias (generales) que no suelen recoger los programas de las distintas asignaturas, y que se deberían potenciar desde el espacio de la tutoría: responsabilidad, autonomía, iniciativa, trabajo en grupo, adaptación al cambio, confianza en si mismo...

La fragmentación que preside los planes de estudio no favorece en absoluto esta visión holista e integrada de la enseñanza. Tan importante es que el alumnado acumule los conocimientos necesarios para el desempeño de una profesión (conocimiento científico), como que sepa moverse y "venderse" en un mundo cada vez más competitivo. Y para ello sí que resulta importante que el estudiantado desarrolle esas otras estrategias y competencias (conocimiento sociopersonal) que muchas veces no están recogidas en los libros y que son contenidos propios del ámbito de la tutoría. Echevarría y col. (1996:207) inciden en esta idea cuando afirman que ".. cada vez más se acepta que en la actividad universitaria se debe implicar a la persona en su conjunto, de modo que el paso de las personas por las instituciones de educación superior suponga una experiencia de crecimiento, no sólo intelectual, sino también social, personal, moral...". En definitiva, la formación universitaria no debe tener por objeto solamente conocimientos y procedimientos, sino también valores, normas y actitudes. Ha de tener por objeto el pleno desarrollo personal del alumnado, un 
desarrollo que implica por parte del profesorado el ejercicio de la función tutorial y orientadora.

\section{DistinTAS MODALIDADES DE TUTORÍA UNIVERSITARIA}

La tutoría es una función que corresponde al docente; enseñar es posibilitar el aprendizaje y para ello se requiere un buen conocimiento del alumnado, sus expectativas, lo que necesita y puede aprender, su estilo de aprendizaje, su motivación, etc. El profesorado en general y el profesor tutor en particular es quien mejor puede ayudar al alumnado a desembarcar y adaptarse a la enseñanza universitaria, a moverse en medio del laberinto que conforman las titulaciones, las asignaturas troncales y optativas, los practicums, los créditos de libre elección, etc. El tutor puede también estimular la promoción académica y profesional del alumnado, asesorándole sobre los estudios de tercer ciclo e informándole sobre las salidas profesionales de cada titulación.

La tutoría universitaria, por tanto, comporta una relación de ayuda, en un clima óptimo y favorable que constituya algo más que la resolución de problemas puntuales y concretos, para extenderse a la dinámica que conforma el proceso formativo en toda su extensión. Como señala Sancho (2002:17) desde una visión amplia, la tutoría universitaria habría que entenderla como "el conjunto de todas las actividades, actitudes, procesos, intercambios personales y profesionales que caracterizan la relación entre el docente y los estudiantes".

Así, frente a una concepción "burocratizada" de la tutoría defendemos una visión amplia de la misma, que vaya mas allá de la tarea que todo profesor universitario realiza en sus asignaturas, abarcando los aspectos que tienen que ver con la madurez y desarrollo integral del alumnado (Lázaro, 2002; García, Fernández y Serra, 2003). Por tanto, abogamos por una tutoría cuyo ámbito de actuación no se restringe puramente a lo formativo, al horario de las 6 horas y al espacio del despacho (tutoría académica), sino que se extiende más allá, haciendo un seguimiento del proceso formativo y estimulando la madurez personal y profesional, con una clara proyección hacia el aprendizaje autónomo, la investigación y el desempeño profesional (tutoría de carrera). Esta otra modalidad de tutoría, que incluye a la primera, se desarrolla en cualquier ámbito que se relacione con los procesos de enseñanza aprendizaje (aula, despacho, seminario...). Dice Almajano (2002:225) que el tutor "tiene como principal función saber escuchar y a partir de lo que oye, ayudar al estudiante a ejercer su libertad, sugiriéndole caminos y sopesando con él para cada uno de ellos, las ventajas y los inconvenientes".

El tutor de carrera será un profesor que se preocupe por la formación integral del alumnado y un referente de éste a lo largo de sus estudios, potenciando su madurez, no sólo como aprendiz sino también como persona y preocupándose además por su proyección social y profesional (Álvarez, 2002). Entre sus funciones podríamos destacar las siguientes:

$\checkmark$ Participar en la elaboración y desarrollo del Plan de Tutorías Universitario (PTU) de su titulación y de su grupo. 
Asesorar a los estudiantes sobre su proceso de adaptación al contexto universitario.

$\checkmark$ Lograr un conocimiento preciso del alumnado que tutela con el fin de ayudarle a resolver las dificultades a las que se enfrenta a lo largo de su carrera.

$\checkmark$ Guiar al alumnado en su proceso de aprendizaje autónomo y las posibilidades académicas y profesionales.

\section{El Plan de Tutorías en la Facultad de Educación de la Universidad de LA LAGUNA}

La necesidad de potenciar la función tutorial del profesorado se planteó en la Universidad de La Laguna desde la implantación de los nuevos planes de estudio y fue recogida en la evaluación sobre la Calidad de los Títulos de maestro especialista (1996-98). En el informe final del proyecto temático "títulos de maestro" correspondiente al programa de evaluación institucional de la calidad de las universidades, se recogieron una serie de puntos débiles detectados, que justificaban la necesidad de impulsar el desarrollo de la función tutorial con el fin de dar respuesta a una serie de carencias encontradas. Concretamente, y en relación a la Docencia, se apreciaron las siguientes debilidades:

Desconocimiento general por parte del alumnado del perfil profesional de las titulaciones y de los objetivos de formación.

$\checkmark$ Desinformación sobre la naturaleza y el carácter de las asignaturas de libre elección e Inadecuada difusión de los programas de las asignaturas.

$\checkmark$ Inadecuada aplicación de los criterios de evaluación de los aprendizajes.

$\checkmark$ Estrategias didácticas inadecuadas.

$\checkmark$ Carencia de información y asesoramiento sobre la especialización profesional.

$\checkmark$ Falta de coordinación entre asignaturas.

$\checkmark$ Incumplimiento del horario de tutorías por parte del profesorado.

El desarrollo de la función tutorial del profesorado se justificó también en base a los datos obtenidos en un diagnóstico de necesidades realizado por el Servicio de Orientación e Información al Alumnado (SOIA) de la Facultad de Educación de la Universidad de La Laguna (Álvarez, 2002). Algunos resultados obtenidos que cabría destacar:

Más del 50\% del profesorado encuestado consideró que el alumnado se hallaba poco o nada informad sobre la titulación y la Facultad en la que estudiaba.

$\checkmark$ Entre un 50 y $65 \%$ del profesorado contestó que el alumnado poseía poca o ninguna información sobre oferta de asignaturas optativas, características de los prácticums o la finalidad de las tutorías académicas.

$\checkmark$ Más del 75\% señaló que el alumnado no poseía información sobre cuáles eran las salidas profesionales de sus estudios. 
$\checkmark$ El 73,2\% del profesorado consideró que el plan tutorial podría mejorar la formación del alumnado si se le considera como una actividad del proceso educativo.

Teniendo en cuenta esta información recogida, en los últimos años se viene desarrollando en la Facultad de Educación un programa de tutorías de carrera para el alumnado universitario. Si bien se pone el énfasis en los estudiantes de primer curso dado que se considera que son quienes necesitan mayor ayuda, la labor tutorial del profesorado se extiende a lo largo de todos los cursos. Con ello se pretende:

1. Facilitar el proceso de adaptación del alumnado a la vida universitaria.

2. Ayudar al alumnado a conocer las características del plan de estudios que ha elegido.

3. Orientar al alumnado en sus estudios y en su proceso de aprendizaje.

4. Orientar al alumnado sobre la toma de decisiones académicas y profesionales.

5. Orientar al alumnado para que se conozca mejor a sí mismo, para que se relacione y para que desarrolle habilidades de cara a su futura inserción laboral.

Para coordinar y dinamizar el desarrollo del programa se crearon tres comisiones de trabajo que realizan sus funciones de manera coordinada:

- Comisión de Tutorías de la Facultad (C.T.F.): dinamiza y supervisa el desarrollo del programa de tutorías, toma acuerdos sobre su desarrollo, etc.

- Comisiones de Tutorías por Titulación (C.T.T.): analiza la realidad y las necesidades de cada titulación, concreta la propuesta de actividades en cada titulación de acuerdo a sus peculiaridades y prioridades, valora los resultados obtenidos, etc.

- Comisión del Programa de Compañero Tutor (C.C.T.): analiza la realidad y las necesidades de cada titulación, planifica acciones adaptadas a la realidad de cada titulación y curso, realiza un seguimiento del desarrollo del programa del compañero tutor etc.

\section{Planificación de la acción: el programa Velero}

Es un programa que está pensado y estructurado para desarrollar la tutoría de carrera, ofreciendo al profesor tutor diferentes propuestas de actividades y recursos. Si bien algunas de las actividades podrían ser desarrolladas por el profesorado en sus horas de atención individualizada (tutoría académica), es una propuesta para el desarrollo global e integral del alumnado, que además de lo formativo, trata lo personal y sociolaboral. Está organizado en torno a 16 actividades. En cada actividad se especifican los siguientes apartados: nombre de la actividad, justificación de su importancia, objetivos concretos que se persiguen, responsable de su puesta en práctica, temporalización, desarrollo de la actividad y recursos.

El programa presenta una secuencia lógica de actividades, pero pueden desarrollarse de manera flexible en función de las necesidades y características del grupo. Es decir, el programa está pensado para que cada tutor seleccione aquellas actividades que considere más apropiadas en función de las características de su grupo. 
La primera parte del programa (actividades 1-3) está pensada para facilitar a comienzo del curso el proceso de adaptación del alumnado a la vida universitaria.

En la segunda parte (actividades 4-6) se afronta la intervención desde una vertiente grupal, intentando que los estudiantes conozcan otros aspectos de la Universidad paralelos a la actividad formativa, participen en otros espacios de la vida universitaria y se cree un ambiente positivo y colaborativo en el grupo clase.

La tercera parte (actividades 7-10) tiene un enfoque más individual de la actuación del tutor y tiene como objetivo ayudar al alumnado a mejorar el conocimiento de sí mismo, de sus expectativas y de las metas que cada uno se plantea.

La cuarta parte del programa (actividades 11-12) busca, después de pasado ya un cuatrimestre, hacer una valoración y seguimiento de la marcha del curso y dar pautas para la mejora del rendimiento académico.

La quinta parte (actividad 13) está pensada para analizar, a través del estudio de casos, aquellas situaciones propias de la vida universitaria que pueden interferir en el proceso formativo del alumnado. Sin embargo, hay que decir que, con frecuencia, la realidad del aula aporta casos reales que se prestan a ser tema de debate, en cuyo caso debemos aprovecharlos ya que el interés y grado de implicación de los alumnos favorece que la dinámica del debate sea más real y enriquecedora.

Por último, la sexta parte (actividades 14-16) constituye un punto y seguido en el proceso de planificación a largo plazo del desarrollo profesional, animando al alumnado a que vaya tomando conciencia de la realidad laboral y estudiando distintas estrategias para afrontar la toma de decisiones.

De forma breve hacemos una presentación de las dieciséis actividades propuestas.

\section{1. "Nos conocemos"}

La llegada a la Universidad supone un momento importante para la vida de todo estudiante. Es un período de incertidumbres y de cambios que hacen necesaria la presencia del tutor y del compañero tutor para ayudarles en este aterrizaje. La acogida en los primeros días de clase y el clima que se cree constituyen aspectos esenciales en los que se va a fundamentar el trabajo que se realizará a lo largo del curso. Se trata de crear un clima de confianza y de conocimiento mutuo entre los distintos miembros del grupo, que favorezca el desarrollo de las distintas actividades formativas que se llevarán a cabo a lo largo del curso.

\section{2. "Despejando dudas"}

Además de la información de carácter general que reciben los estudiantes acerca de la Universidad antes de comenzar los estudios, una vez dentro es importante que conozcan una serie de aspectos relacionados con la titulación que han escogido y con el centro, donde a partir de ese momento van a pasar una parte considerable de su tiempo. Para ello se organizan las Jornadas de Bienvenida, cuya finalidad es informar de las características de la carrera, servicios universitarios, fórmulas de participación, etc.. 


\section{3. "Conocemos nuestro entorno"}

Dentro del proceso de adaptación a la enseñanza superior, es fundamental que el alumnado conozca la ubicación de los servicios e instituciones a las que deberá acudir para solicitar o resolver cuestiones que tienen que ver con su estancia en la Universidad.

\section{4. "La otra cara de la universidad"}

Lograr la presencia y participación de los estudiantes en los órganos donde tienen cabida, es un reto y un objetivo compartido por todas las instituciones universitarias. A esto hay que unir, la participación en otros foros formativos o lúdicos que complementan la formación, donde el alumnado podrá desarrollar algunas habilidades sociales y capacidades que se están demandando en el mundo laboral.

\section{5. "Ser estudiante universitario"}

El tránsito a la Universidad supone un cambio importante y es preciso que el alumnado tome conciencia de dicho cambio y de las nuevas condiciones en las que se desarrolla ahora el proceso formativo, bastante diferente al de etapas anteriores. En juego está su propia adaptación al contexto universitario y los logros futuros (académicos - profesionales) que cada uno pueda conseguir.

\section{6. "Aprendiendo a trabajar en grupo"}

Lograr la cohesión dentro del grupo clase, creando los hábitos necesarios y desarrollando las capacidades adecuadas constituye un elemento básico para lograr una buena participación del alumnado en las distintas actividades. Es un aprendizaje individual y/o colectivo, a través del cual cada uno de los estudiantes va desarrollando actitudes y capacidades de trabajo en equipo.

\section{7. "Ser uno mismo"}

Debería propiciarse que el alumnado tomara conciencia de las características personales, con el fin de que se produzca un adecuado ajuste entre sus expectativas y logros. Cada persona tiene unas cualidades determinadas y es fundamental que cada uno acepte su individualidad; que se valore tal como es para que a partir de ahí establezca con claridad el marco de las metas a conseguir.

\section{8. "Mis expectativas"}

Es importante que los estudiantes reflexionen sobre distintos aspectos que tienen que ver con el proceso formativo y con las expectativas respecto a su futuro. Es por tanto necesario que se valoren dichas expectativas, con el fin de detectar posibles desajustes que pueden suponer un bloqueo de cara a la consecución de las metas que se propongan.

\section{9. "Dialogando"}

Se trata de ayudar al alumnado a valorar determinadas cualidades personales que pueden resultar importantes para su desarrollo personal y profesional. A través de la 
conversación entre dos conceptos antagónicos, se puede llegar a que cada uno se conozca, se valore mejor a sí mismo y reflexione sobre su manera de ser y de actuar.

\section{0. "Cara a cara"}

Las entrevistas personales sirven para que el tutor ayude a los estudiantes a valorar sus características, sus actitudes, sus dificultades, sus expectativas, etc. El contacto personal puede favorecer la comunicación y permitir que el alumnado exprese sus inquietudes, preocupaciones y percepciones acerca de la realidad, sin la presión que puede ejercer su grupo de iguales.

\section{1. "Clima del aula"}

Conocer de qué manera el alumnado se sitúa en relación al grupo clase y cómo percibe el desarrollo de los procesos educativos, ha de constituir un elemento importante para tomar decisiones sobre la enseñanza que se imparte. A partir de los resultados se puede iniciar una revisión y reflexión entre todo el profesorado que imparte docencia al grupo, acerca de las condiciones y características del modelo educativo que se está desarrollando, con el fin de mejorar posibles desajustes y adecuarlo a las necesidades del alumnado .

\section{2. "Estudiamos con sentido"}

Se pretende ofrecer, con un carácter preventivo, algunas pautas básicas para optimizar el proceso de estudio y de aprendizaje del alumnado. La llegada a la Universidad coincide con muchos cambios en cuanto a metodología didáctica, por lo que sin un asesoramiento adecuado muchos estudiantes tienen dificultades para adaptarse a los ritmos, exigencias y dinámica de los procesos instructivos. El conocimiento y aplicación de algunas estrategias de estudio puede facilitar enormemente el proceso de enseñanza aprendizaje. Dado que existe una gran cantidad de procesos mentales que están presentes en las actividades que diariamente realiza el estudiante (tomar apuntes, estructurar la información, analizar textos, etc.), es por lo que las técnicas de estudio y de aprendizaje constituyen un salvoconducto importante para realizar de manera conveniente las tareas académicas y lograr el éxito académico.

\section{3. "Investigando nuestra dudas"}

La vida universitaria entraña numerosas dificultades de muy distinta naturaleza que los estudiantes han de resolver. Conocer algunos procedimientos básicos para hacer frente a dichas situaciones es un modo de prevenir y de prepararse para ser capaces de poner en marcha las estrategias adecuadas para resolver cada dificultad.

\section{4. "Explorando mis cualidades"}

Antes de tomar decisiones sobre el futuro profesional, es necesario que cada alumno se detenga a pensar en sus propias cualidades y características personales. El estudio de los rasgos definitorios de la personalidad de cada uno ha de ser el soporte en el que se fundamenten las decisiones que se tomen, tratando de encontrar el óptimo equilibrio entre capacidades y oportunidades; entre características y posibilidades. Las metas de carrera que se tracen y la confección del proyecto pro- 
fesional de cada persona deberían estar iluminadas, sobre todo, por una toma de conciencia de los rasgos que le definen. Construir un proyecto profesional es seleccionar objetivos a largo plazo, definir lo que quiere hacerse, calcular las posibilidades, especificar las necesidades y valorar las dificultades que se pueden encontrar en el camino,

\section{5. "Tomando decisiones"}

Una habilidad importante que debe potenciarse en cualquiera de los niveles educativos y, como no, en el contexto de la enseñanza universitaria, es la de resolver problemas y saber elegir entre aquellas opiones que mejor se adapten a sus intereses y posibilidades. Tomar decisiones es una tarea compleja por la trascendencia que tiene en el desarrollo de la carrera de cada persona. Por ello hay que entrenar a los estudiantes en el manejo de estrategias y procedimientos que aseguren la coherencia y adecuación de las decisiones que se adopten.

\section{6. "Pensando en la profesiones"}

En los primeros años de los estudios universitarios, el alumnado debe empezar a pensar en la planificación de su carrera profesional; sin prisa, pero sin pausa. Es necesario que comience a tomar conciencia de la realidad laboral que presenta el área a la que se intentará incorporar en el futuro, de tal modo que la configuración de su itinerario formativo esté también iluminado por esta información ocupacional. Se trata de despertar la motivación, la curiosidad y el interés por su futuro profesional con una perspectiva de prospección y con la vista puesta en el futuro.

\section{Desarrollo y Evaluación del Programa}

Para valorar en qué medida se habían cumplido los objetivos establecidos en el programa, se llevó a cabo en el curso 2003-2004 un proceso de evaluación con los estudiantes y tutores que habían participado en el desarrollo del mismo. Con ello se pretendía evaluar los logros alcanzados y poder tomar decisiones en relación a los cambios o mejoras a introducir en los cursos siguientes (Sanz, 1990; Stufflebean y Shinkfield, 1995).

Para conocer la opinión del alumnado se aplicó un cuestionario ad hoc al que respondieron 145 estudiantes de primer curso de la Facultad de Educación de la Universidad de La Laguna que habían participado en el programa. Los sujetos encuestados pertenecían a las titulaciones de Pedagogía, Maestro de Educación Física, Maestro de Educación Infantil y Maestro de Educación Primaria.

De los estudiantes que respondieron al cuestionario, un 74,1\% participó en más de dos sesiones del programa de actividades tutoriales y consideraron que la labor realizada por el tutor/a que tuvieron asignado fue buena $(63,4 \%)$ o muy buena $(28,3 \%)$. En general, el alumnado valoró positivamente el programa, señalando que fue adecuado $(62,1 \%)$ o muy adecuado $(18,6 \%)$, puesto que les ayudó a adaptarse a la vida universitaria y a conocer mejor la Universidad. Asimismo, el 63,4\% señaló que las actividades realizadas les habían ayudado a lograr un conocimiento más preciso de sus características, de sus posibilidades y de las salidas profesionales de 
la titulación que se encontraban cursando. En relación a la tutoría académica, por lo general, no es un recurso muy utilizado por el alumnado, a pesar de que un 48,4\% señaló que había acudido alguna vez a las horas de tutoría de las asignaturas, sobre todo antes de los exámenes (31,5\%).

Destacar, no obstante, que el 48,3\% del alumnado indicó que las actividades del programa no le habían ayudado a mejorar el rendimiento en las asignaturas que habían cursado. Si bien es un dato a valorar con cautela, lo que si es cierto que el profesor tutor tiene que lograr que las actividades tutoriales se vinculen a la práctica educativa y que reviertan en el proceso de aprendizaje del alumnado. No se pueden desligar los distintos tipos de acciones; por el contrario las actividades tutoriales deben verse como una parte más de la labor educadora y como un apartado complementario de la labor docente en la línea de las propuestas de la convergencia europea.

Como principales aspectos negativos el alumnado señaló que el programa comenzó a desarrollarse cuando ya estaba iniciado el curso, y que el horario destinado a las tutorías era inadecuado.. Si bien se ha conseguido implantar una hora semanal en el horario académico, en algunos casos como Pedagogía se ha situado en la última hora de la mañana o en la primera hora de la tarde, lo que dificulta la asistencia a las distintas actividades. Por eso vemos necesario ubicar el tiempo de las tutorías en franjas intermedias del horario normal de clase. En ese sentido se debe plantear en las Comisiones de curso de las distintas titulaciones de la Facultad la conveniencia de ubicar el tiempo de las tutorías en huecos intermedios del horario.

De las 16 actividades del programa, "Nos conocemos" y "Ser estudiante universitario" fueron las que realizaron todos los estudiantes. El resto de las actividades no fueron desarrolladas de manera generalizada, sino que cada tutor escogió aquellas que estimó más oportunas en función de las características del grupo.

Las valoraciones del profesorado se obtuvieron a través de un cuestionario ad hoc que fue cumplimentado por los 12 tutores que Ilevaron a cabo labores de tutela con estudiantes de primer curso. Excepto la 14, 15 y 16, el resto de las actividades fueron puestas en práctica al menos por un tutor de los que respondieron al cuestionario.

Las actividades 2, 11 y 12 ("Despejando dudas"; "Clima del aula" y "Estudiamos con sentido") fueron puestas en práctica por 8 tutores, mientras que las actividades 4, 6, 7, 8 y 13 ("La otra cara de la universidad"; "Aprendiendo a trabajar en grupo"; "Ser uno mismo"; "Mis expectativas" e "Investigando nuestras dudas") las desarroIlaron 5 tutores. Por último comentar que algunos tutores han Ilevado a la práctica algunas otras actividades no contempladas en la guía, básicamente referidas a debates sobre distintos temas de interés para el alumnado: Evaluación, relación con compañeros, comunicación en el aula, relación profesor-alumnos, actividades de convivencia con alumnos fuera de la universidad, etc.

\section{CONCLUSIONES}

El modelo de tutorías que se viene desarrollando en la Facultad de Educación y los resultados que se vienen obteniendo con el mismo nos hacen mirar el futuro con optimismo. Los estudiantes que han participado en el plan tutorial han valorado de 
manera positiva la existencia del programa, así como las actividades desarrolladas. Consideran que el programa de tutorías es un elemento importante del proceso formativo, puesto que les permite contar con un profesor/a que les apoya y al que pueden acudir en el momento en el que lo necesiten para resolver dudas y tomar decisiones. Asimismo estiman que la tutoría facilita la adaptación a la Universidad y posibilita la reflexión sobre lo que significa ser estudiante universitario. Valoran positivamente la actitud del profesorado y el interés por vincular los aprendizajes del aula con esa otra esfera de lo personal y profesional, que entraña la formación como persona y como futuro trabajador dentro del amplio campo de la educación.

Es importante esta valoración positiva, puesto que la percepción inicial que tienen de la formación en su primer año de estudios universitarios y del contexto en el que van a pasar una parte importante de su tiempo, puede constituir un elemento clave en el que se han de fundamentar otros logros futuros. El sentir desde el principio que cuentan con la ayuda de una persona que les puede servir de referencia, constituye sin duda un elemento importante para prevenir situaciones de inseguridad que pueden conducir al fracaso o al abandono de los estudios. Recomiendan que de cara a los próximos cursos se lleven a cabo las acciones que permitan una mayor participación del alumnado en las tutorías y que el programa comience desde el inicio del curso.

Por su parte, los profesores tutores hacen también una valoración positiva de la labor realizada, puesto que las actividades desarrolladas, tanto en horas de clase como en otros momentos que se han aprovechado para asesorar al alumnado, han servido para alcanzar un conocimiento más ajustado de las características de éstos y para ayudarles a reflexionar sobre su vida académica actual y su vida profesional futura.

\section{Bibliografía}

ALMAJANO, P. (2002). Experiencias previas de tutoría. En Coriat, M. (ed). Jornadas sobre tutorías y orientación. Granada: Editorial Universidad de Granada, 223-236. ÁlVAREZ, P. y CABRERA, L. (1998). El Servicio de Orientación e Información al Alumnado (SOIA): un proyecto innovador en el C.S.E. de la ULL. Congreso sobre Orientación Universitaria. Barcelona: Universidad de Barcelona.

ÁLVAREZ, P., CABRERA, L., FELICIANO, L. y SANTANA, L. (1999). Intervención psicopedagógica en el ámbito universitario: valoración de una experiencia en el centro superior de educación de la ULL. REOP, 10 (18), 379-387.

ÁlVAREZ, P., BETHENCOURT, J. y CABRERA, L. (2000). La transición al mercado laboral de los psicopedagogos: estudio de las dos primeras promociones en la Universidad de La Laguna. Revista de Psicología General y Aplicada, 53 (3), 535-547. ÁLVAREZ, P. (2002). La función tutorial en la Universidad; una apuesta por la mejora de la calidad de la enseñanza. Madrid EOS.

ÁLVAREZ, P. y JIMÉNEZ, H. (comp.) (2003). Tutoría Universitaria. Tenerife: Servicio de Publicaciones de la ULL.

ÁLVAREZ, V. y LÁZARO, A. (2002). Calidad de las universidades y orientación Universitaria. Málaga: Aljibe. 
ARBIZU, F. (1994). La labor orientadora del profesor universitario desde la perspectiva del alumnado y el profesorado. Revista de Investigación Educativa, 23, 614-622.

CASTELLANO, F. y SANZ, R. (1990). Un análisis de necesidades entre estudiantes de la Universidad de Granada para la elaboración de un programa de orientación educativa. Revista de Investigación Educativa, 8 (16), 149-155.

DÍAZ, T. (1989). La orientación universitaria ante la problemática académica y profesional del estudiante. Madrid: Narcea.

ECHEVARRÍA, B., FIGUERA, P. y GALLEGO, S. (1996): La Orientación universitaria: De sueño la realidad. Revista de Orientación y Psicopedagogía, 7 (12), 207-220.

GARCÍA, E., FERNÁNDEZ, A. y SERRA, B. (2003). La formación integral de los alumnos a través de los profesores tutores: una experiencia de la Universidad Politécnica de Valencia. En Álvarez, P. y Jiménez, H. (comp.). Tutoría universitaria. Tenerife: Servicio de Publicaciones de la Universidad de La Laguna, 245-255.

LÁZARO, A. (2002). La acción tutorial en la función docente universitaria. En Álvarez, V. y Lázaro, A. Calidad de las universidades y orientación universitaria. Málaga: Aljibe, 249-282.

MARTÍN, M., MORENO, E. y PADILLA, T. (1998). La orientación para el acceso a la Universidad: análisis de las necesidades expresadas por una muestra de estudiantes de nuevo ingreso. Revista Española de Orientación y Psicopedagogía, 9 (16), 257-271.

SALDAÑA, L. (1986). Éxitos y fracasos en la Universidad. En Latiesa, M. (comp.). Demanda de Educación Superior y rendimiento académico en la universidad. Madrid: MEC.

SANCHO, J. (2002). El sentido y la práctica de las tutorías de asignaturas en la enseñanza universitaria. En Coriat, M. (ed). Jornadas sobre tutorías y orientación, Granada: Editorial Universidad de Granada, 17-36.

SANZ, R. (1990). Evaluación de programas de Orientación Educativa. Madrid: Pirámide.

STUFFLEBEAN, D. y SHINKFIELD, A. (1995). Evaluación Sistemática. Guía teórica y práctica. Barcelona: Paidos. 